\title{
Bilateral Hypoplasia of the Medial and Lateral Menisci
}

\author{
Yoon-Seok Youm, MD, Sung-Do Cho, MD, Hye-Yong Cho, MD, and Tae-Wook Kang, MD \\ Department of Orthopedic Surgery, Ulsan University Hospital, University of Ulsan College of Medicine, Ulsan, Korea
}

Hypoplasia of the meniscus is a very rare congenital abnormality, with only a few cases reported to date. A 9-year-old girl visited our hospital due to lateral knee pain following a hyperextension injury to the left knee. Magnetic resonance imaging showed hypoplasia of the medial and lateral menisci, as well as a posterior horn tear of the lateral meniscus, in both knee joints. To our knowledge, this is the first report of a patient with hypoplasia of the medial and lateral menisci in both knee joints.

Keywords: Knee, Meniscus, Hypoplasia

Discoid and abnormally attached menisci are common congenital abnormalities of the meniscus ${ }^{1,2)}$. In contrast, hypoplasia of the meniscus is very rare. To date, it has been reported alone $e^{3-6)}$, associated with other abnormalities of the knee joint ${ }^{5,7,8)}$, and in patients with partial deficiency of the meniscus ${ }^{9)}$. To our knowledge, however, there have been no reports describing hypoplasia of the medial and lateral menisci in both knee joints.

\section{Case Report}

A 9-year-old girl visited our hospital due to lateral knee pain that developed one week after a hyperextension injury of her left knee joint during exercise. Physical examination revealed lateral joint line tenderness and positive results for the McMurray test in both knee joints. The Lachman test, anterior and posterior drawer and pivot shift tests, and maneuvers for rotator instability were all negative. The range of motion (ROM) was no flexion

Received November 20, 2015; Revised January 5, 2016;

Accepted February 26, 2016; Published Online: April 25, 2017

Correspondence to: Sung-Do Cho, MD

Department of Orthopedic Surgery, Ulsan University Hospital, University of Ulsan College of Medicine, 877 Bangeojinsunhwando-ro, Dong-gu, Ulsan 44033, Korea

Tel: +82-52-250-7129, Fax: +82-52-235-2823

E-mail: sdcho@uuh.ulsan.kr

This is an Open Access article distributed under the terms of the Creative Commons Attribution Non-Commercial License (http://creativecommons.org/licenses/by-nc/4.0/) which permits unrestricted non-commercial use, distribution, and reproduction in any medium, provided the original work is properly cited. contracture and a further flexion of $120^{\circ}$ with mild pain in her left knee. Plain radiographs showed no specific findings and the notch width index was 0.234 .

Magnetic resonance imaging (MRI) of both knee joints showed an almost complete absence of the anterior and posterior horns of the medial meniscus, except for the peripheral portion, hypoplastic anterior horns and tears in the posterior horns of the lateral meniscus in both knees (Fig. 1). The patient did not complain of pain or discomfort in her right knee, but did complain of pain and limited motion in her left knee. We, therefore, decided to perform arthroscopic surgery only on her left knee.

At arthroscopy, the medial meniscus showed hypoplastic changes (approximately $5 \mathrm{~mm}$ in width and $1-2 \mathrm{~mm}$ in height). The anterior horn of the lateral meniscus showed hypoplastic changes, while the posterior horn was connected to the meniscofemoral ligament with fibrous tissue. This fibrous tissue extended and covered the popliteal hiatus. Both the anterior and posterior cruciate ligaments were intact and there was no chondral pathology medially or laterally. Arthroscopic partial meniscectomy was performed for the horizontal tear of the posterior horn of the lateral meniscus (Fig. 2).

Postoperatively, the patient was allowed to bear weight and perform knee motions as tolerated. Two weeks after the operation, she restored full active and passive ROM without pain. At 4-year follow-up, she had no symptoms or abnormal objective findings for both knees and she had returned to full activity. On the follow-up MRI for both knees at postoperative 4 years, there were no chondral lesions or tears of the remnant meniscus in the left 

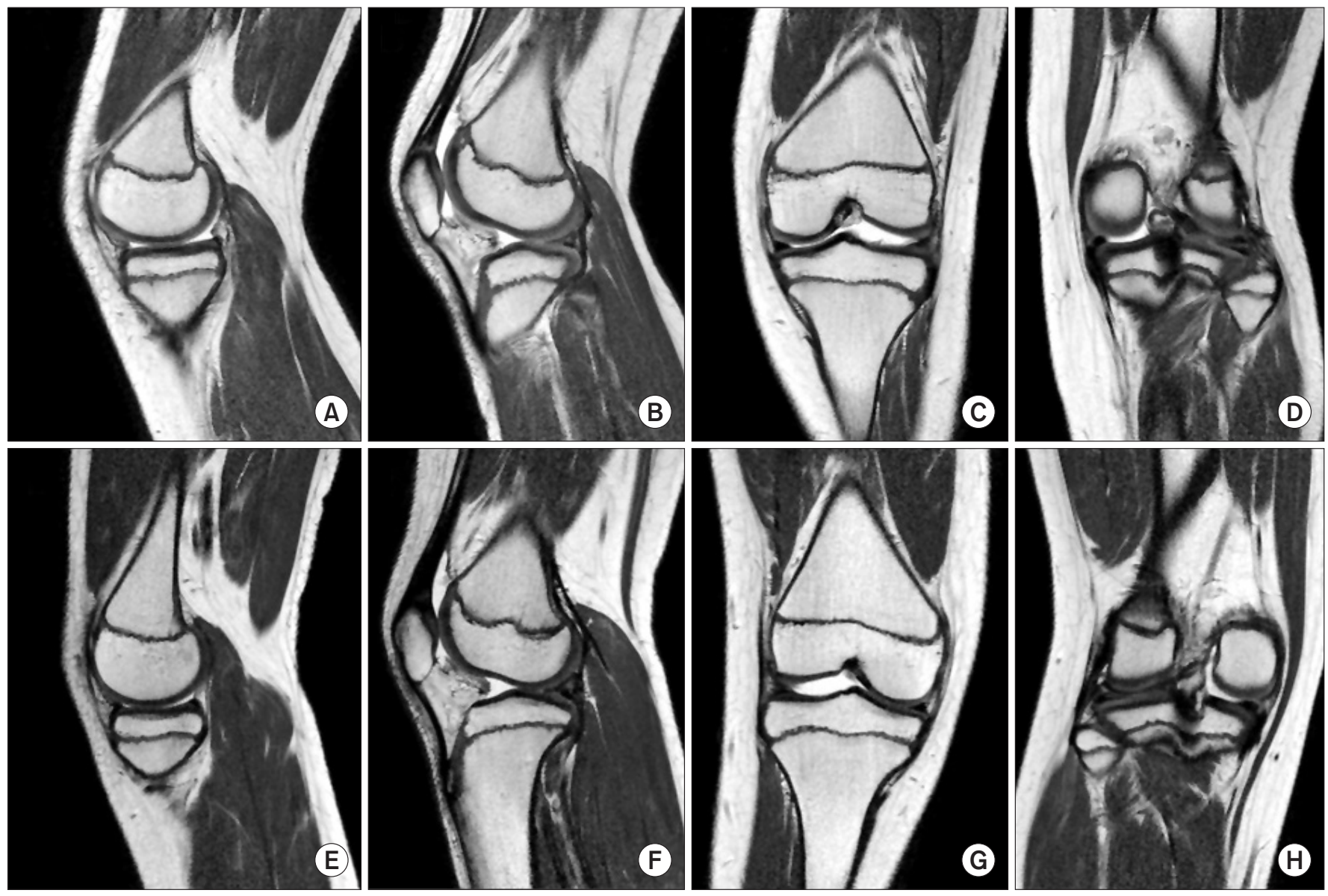

Fig. 1. Magnetic resonance imaging of the left $(\mathrm{A}-\mathrm{D})$ and right $(\mathrm{E}-\mathrm{H})$ knee joints. (A, E) Hypoplastic anterior and posterior horns of the medial menisci. (B, F) Hypoplastic anterior horns and posterior horn tears of the lateral menisci. (C, D, G, H) Hypoplasia of both menisci and lateral meniscus tears.

knee and no significant changes in the right knee.

\section{Discussion}

The characteristic shapes of both the medial and lateral menisci are observed early in prenatal development, maturing along with the capsule and coronary and cruciate ligaments. No abrupt changes in development occur after birth. Rather, gradual changes occur, including a decrease in vascularity, progressing from the center to the peripheral margins; a growth in size; and changes in the configuration of the menisci $i^{1)}$. The association of other anomalies in the knee with hypoplastic menisci may be due to the common mesenchymal origin of these structures ${ }^{1,2)}$. However, in the patient described here, the only anomalies were in the menisci.

The most common congenital abnormality of the meniscus is a discoid meniscus, which is more frequent in the lateral than the medial meniscus ${ }^{1,5)}$. Other abnormalities have been reported, including abnormal attachments of the meniscus, hypoplasia and partial deficiency ${ }^{3-9)}$. These abnormalities were all incidentally diagnosed in patients with symptoms associated with trauma or other congenital anomalies. Thus, their exact incidence is unknown $^{2,3)}$.

Congenital hypoplasia of the medial meniscus has been reported in three patients ${ }^{4,6)}$, and bilateral hypoplasia of the medial meniscus with an Outerbridge grade III chondral lesion of the medial femoral condyle in one patient $t^{2}$. One patient was reported to have partial deficiency of the lateral meniscus ${ }^{9)}$ and another was found to have bilateral congenital absence of the anterior cruciate ligament and the internal menisci of the knee ${ }^{7}$. Another patient was found to have congenital absence of both menisci and both cruciate ligaments with radial club hands and congenital thrombocytopenia $^{5}$. Bilateral hypoplastic lateral menisci with an osteochondral lesion in the lateral tibial plateau were reported in one patient ${ }^{3}$, with another having congenital hypoplasia of the lateral meniscus and anterior cruciate ligament associated with osteochondritis dissecans of the medial femoral condyle ${ }^{8}$. Osteochondritis dissecans of the medial femoral condyle associated with 

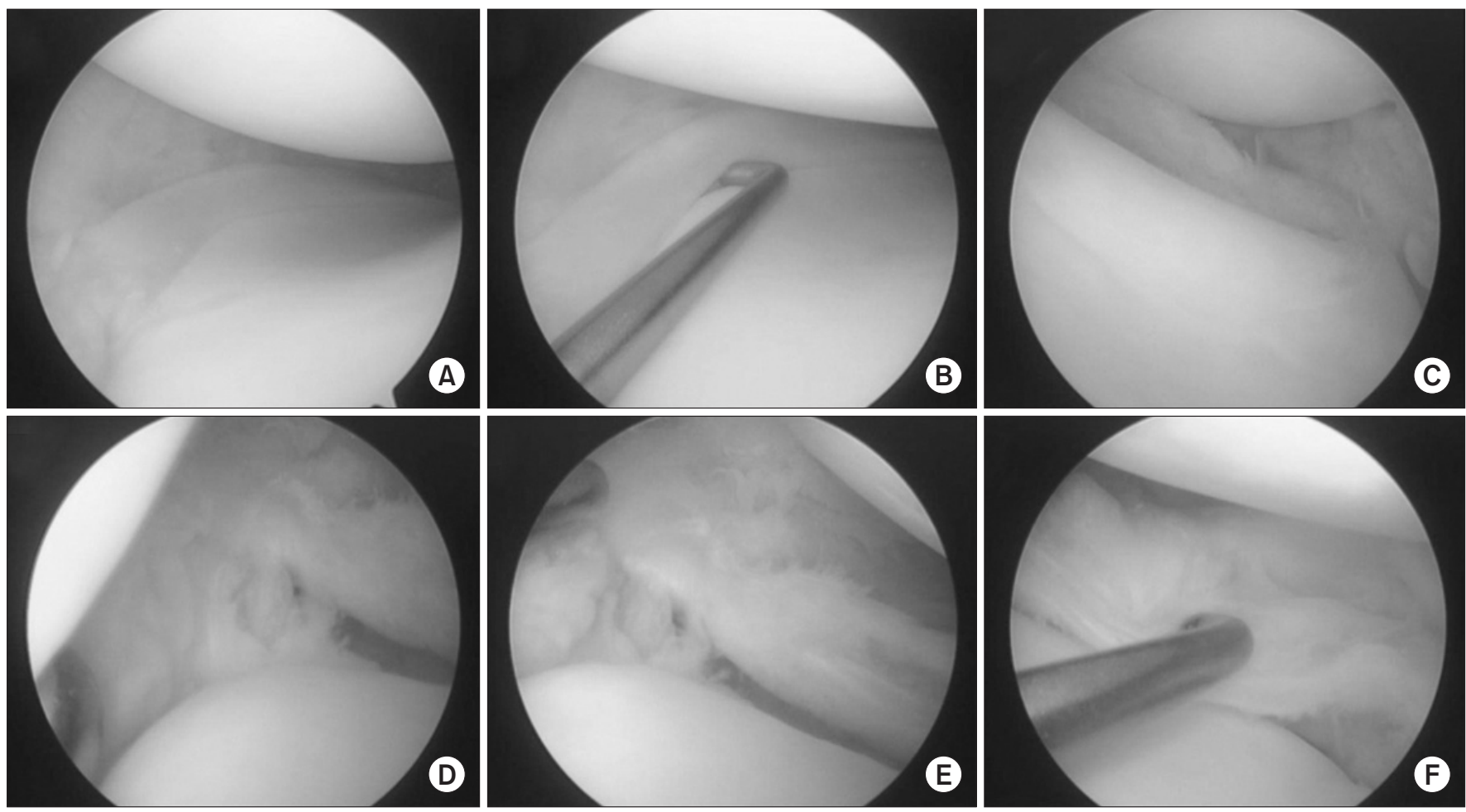

Fig. 2. Arthroscopic findings of the left knee joint. (A, B) Hypoplastic anterior and posterior horns of the medial meniscus. (C) Hypoplastic anterior horn of the lateral meniscus. (D, E) Abnormal attachment of the posterior horn of the lateral meniscus. The posterior horn was connected to a meniscofemoral ligament with fibrous tissue. (F) Extension of the fibrous tissue covering the popliteal hiatus.

hypoplastic medial and partially deficient lateral menisci was also reported $^{10)}$. However, we have been unable to find a description of bilateral hypoplastic medial and lateral menisci. We think that future research efforts are necessary to detect the incidence and clinical course in patients with hypoplastic menisci.

\section{Conflict of Interest}

No potential conflict of interest relevant to this article was reported.

\section{References}

1. Clark CR, Ogden JA. Development of the menisci of the human knee joint: morphological changes and their potential role in childhood meniscal injury. J Bone Joint Surg Am. 1983;65:538-47.

2. Monllau JC, Gonzalez G, Puig L, Caceres E. Bilateral hypoplasia of the medial meniscus. Knee Surg Sports Traumatol Arthrosc. 2006;14:112-3.

3. Ohana N, Plotquin D, Atar D. Bilateral hypoplastic lateral meniscus. Arthroscopy. 1995;11:740-2.
4. Pfeil E. The hypoplastic, hypermobile meniscus. Beitr Orthop Traumatol. 1967;14:3-5.

5. Tolo VT. Congenital absence of the menisci and cruciate ligaments of the knee: a case report. J Bone Joint Surg Am. 1981;63:1022-4.

6. Twyman RS, Ferris BD. Congenital hypoplasia of the medial meniscus: a report of two cases. Arthroscopy. 1991;7:148-50.

7. Dejour H, Neyret P, Eberhard P, Walch G. Bilateral congenital absence of the anterior cruciate ligament and the internal menisci of the knee: a case report. Rev Chir Orthop Reparatrice Appar Mot. 1990;76:329-32.

8. Mitsuoka T, Horibe S, Hamada M. Osteochondritis dissecans of the medial femoral condyle associated with congenital hypoplasia of the lateral meniscus and anterior cruciate ligament. Arthroscopy. 1998;14:630-3.

9. Tetik O, Doral MN, Atay OA, Leblebicioglu G, Turker S. Partial deficiency of the lateral meniscus. Arthroscopy. 2003; 19:E42.

10. Beyzadeoglu T, Gokce A, Bekler H. Osteochondritis dissecans of the medial femoral condyle associated with malformation of the menisci. Orthopedics. 2008;31:504. 